\title{
Temperature dependent carrier lifetime studies on Ti-doped multicrystalline silicon
}

\author{
B. B. Paudyal, ${ }^{1, \text { a) }}$ K. R. McIntosh, ${ }^{1}$ and D. H. Macdonald ${ }^{2}$ \\ ${ }^{1}$ Centre for Sustainable Energy Systems, Australian National University, Canberra, Australian Capital \\ Territory 0200, Australia \\ ${ }^{2}$ Department of Engineering, Australian National University, Canberra, Australian Capital Territory 0200, \\ Australia
}

(Received 2 February 2009; accepted 26 April 2009; published online 23 June 2009)

\begin{abstract}
Carrier lifetime measurements were performed on deliberately Ti-doped multicrystalline silicon wafers using a temperature controlled photoconductance device. The dominant recombination center was found to be the double-donor level associated with interstitial titanium. The interstitial Ti concentrations in multicrystalline silicon wafers were determined by measuring the ShockleyRead-Hall time constant for holes and using the known values of the thermal velocity and capture cross section for holes of the double-donor level at different temperatures. The measured values of the $\mathrm{Ti}$ concentration were then used to determine the electron capture cross section of the double-donor level over the temperature range of $140-270{ }^{\circ} \mathrm{C}$ via the measured values of the Shockley-Read-Hall time constant for electrons and the known thermal velocity. Multiphonon emission was found to be the most likely capture mechanism for this temperature range for electron capture into the double-donor level of Ti in silicon. The effective segregation coefficient for Ti was estimated by fitting Scheil's equation to the measured values of the Ti concentrations and their respective vertical positions in the ingot. If all $\mathrm{Ti}$ were present as the interstitial double-donor, a lower limit of $1.8 \times 10^{-6}$ can be ascribed to the segregation coefficient, which is very close to the equilibrium value. () 2009 American Institute of Physics. [DOI: 10.1063/1.3139286]
\end{abstract}

\section{INTRODUCTION}

Titanium (Ti) can occur as a grown-in impurity during silicon ingot growth, especially in applications for photovoltaics. ${ }^{1}$ It can also contaminate silicon devices during fabrication, such as during sputtering or by mechanical contact in the production line. ${ }^{2}$ Once incorporated in a device, in most cases Ti remains dissolved in silicon due to its low diffusivity. Ti incorporates into the silicon lattice in interstitial form, introducing one of three defect energy levels ${ }^{2}\left(E_{t}\right)$. These defect levels are, namely, an acceptor, a donor, and a double donor, all of which can act as recombination centers.

The electronic properties of the Ti defect levels in silicon have been studied by several groups, as summarized by Graff, $^{2}$ with most of them based on the deep-level transient spectroscopy (DLTS). The defect energy levels have been determined by DLTS to be $E_{c}-0.08, E_{c}-0.27$, and $E_{v}$ +0.28 for the accepter, donor, and double donor, respectively, where $E_{c}$ and $E_{v}$ represent the energy level of the conduction and valance bands in silicon. These defect energy levels were determined at a particularly low temperature $(<200 \mathrm{~K})$; however the common assumption is that the invariable nature of defect energy level with temperature. DLTS has also been employed to determine the electron capture cross section $\sigma_{n}(T)$ for the acceptor and donor defects and the hole capture cross section $\sigma_{p}(T)$ for the double-donor defect, each as a function of temperature. ${ }^{2}$

In addition to DLTS, lifetime spectroscopy has been used to characterize the Ti defect. Rein ${ }^{3}$ employed a mixture

a)Electronic mail: bijaya.paudyal@anu.edu.au. of injection dependent, doping dependent, and temperature dependent lifetime spectroscopies to determine $E_{t}$ and the ratio of the capture cross sections $\left(\sigma_{n} / \sigma_{p}\right)$, also known as the symmetry factor $k$. More recently, Roth et al. ${ }^{4}$ performed temperature dependent lifetime spectroscopy (TDLS) with injection dependent lifetime spectroscopy (IDLS) with photoluminescence lifetime measurements to determine $E_{t}$ and $k$. The value of $E_{t}$ as determined by Rein $\left(E_{v}\right.$ $+0.289 \pm 0.005 \mathrm{eV}$ ) has close agreement with the doubledonor level from DLTS. ${ }^{2}$ One value of $E_{t}$ determined by Roth et al. $\left(E_{c}-0.24 \pm 0.03 \mathrm{eV}\right)$ has fair agreement with the donor level from DLTS; ${ }^{2}$ however their second energy level $\left(E_{c}-0.49 \pm 0.03 \mathrm{eV}\right)$ does not match any levels detected by DLTS. ${ }^{2}$ Note that both Rein ${ }^{3}$ and Roth et al. ${ }^{4}$ calculated $\sigma_{n}$ at room temperature from $k$ assuming the $\sigma_{p}$ at $200 \mathrm{~K}$ listed by Graff. $^{2}$

This paper presents temperature and injection dependent lifetime measurements performed on deliberately Ticontaminated, directionally solidified $p$-type multicrystalline silicon wafers. ${ }^{5,6}$ Samples were selected from different parts of the ingot and were measured in a temperature controlled inductive coil photoconductance apparatus, as discussed in Sec. II. In Sec. III, we present the analysis of the measured lifetime data to confirm the dominance of the double-donor level of $\mathrm{Ti}$ in the wafers and to determine the interstitial $\mathrm{Ti}$ concentration $N_{t}$ for each wafer. We then determine $T$-dependent values of $\sigma_{n}(T)$ for the double-donor level and analyze this dependence in terms of the likely carrier capture mechanism. Finally, the paper presents a lower limit for the effective segregation coefficient for Ti in directionally solidified multicrystalline. 


\section{EXPERIMENT}

Four Ti-contaminated boron-doped multicrystalline silicon wafers were selected for this experiment. Large-area wafers $\left(12 \times 12 \mathrm{~cm}^{2}\right)$ were taken from $14 \%, 37 \%, 53 \%$, and $81 \%$ below the top of the ingot to enable the segregation of $\mathrm{Ti}$ along the ingot length to be studied. The nominal resistivity of the samples was $1.0 \Omega \mathrm{cm}$; however the boron concentrations on each samples were determined from the dark conductance using the carrier mobility model developed by Reggiani et al. ${ }^{7}$ at $300 \mathrm{~K}$; it was found to vary slightly due to dopant segregation during crystallization. The concentration of $\mathrm{Ti}$ added to the silicon prior to commencement of crystallization $^{5,6}$ was 10 ppmw, which equates to approximately $2.9 \times 10^{17} \mathrm{~cm}^{-3}$. Furthermore, one control wafer was selected from the middle of another ingot prepared by the same procedure with similar boron doping but without $\mathrm{Ti}$ contamination in order to verify the dominance of the $\mathrm{Ti}$ defect on the lifetime measurements.

All wafers were etched and cleaned prior to a phosphorus gettering step, which removes fast diffusing interstitial metals such as Fe, which could otherwise partially mask the impact of $\mathrm{Ti}$ on the carrier lifetime. After removal of the gettering layers and further cleaning, plasma-enhanced chemical vapor-deposited silicon nitride films were applied to both surfaces, which acts to suppress surface recombination. Quasi-steady-state photoconductance ${ }^{8}$ (QSSPC) measurements were performed at room temperature on one wafer to identify the region that had the highest and most uniform carrier lifetime and that also fits the heating and cooling stages of the measurement device. This same section (3.5 $\times 3.5 \mathrm{~cm}^{2}$ ) was cut out of each wafer for subsequent analysis. This was necessary to ensure that the recombination is dominated by $\mathrm{Ti}$ and not by crystallographic defects such as dislocation clusters.

Lifetime measurements were performed with a temperature controlled inductive coil photoconductance measurement device detailed elsewhere. ${ }^{9}$ The measurement temperature range has since been extended to $300{ }^{\circ} \mathrm{C}$. The lifetime measurement technique itself is the well established QSSPC technique. ${ }^{8}$ The subsequent analysis employed the carrier mobility model developed by Reggiani et al., ${ }^{7}$ which states how the mobility in equilibrium depends on temperature and dopant concentration. It was modified to account for the injection of excess carriers $\Delta n$ by replacing the donor $N_{D}$ and acceptor $N_{A}$ concentrations with $N_{D}+\Delta n$ and $N_{A}+\Delta n$. When $\Delta n \leq N_{A}$ (or $\left.N_{D}\right)$, this approximation was found to give good agreement with Klaassen's mobility model, ${ }^{10,11}$ which does account for $\Delta n$. Reggiani's model was preferred to Klaassen's in this case because of its larger temperature range.

The temperature across the wafer was found to vary by $2 \%$ during measurement, and the uncertainty in lifetime was $\pm 6 \%,{ }^{9}$ which mostly depends on the calibration of the illumination intensity and inductive coil. The effective lifetime $\tau_{\text {eff }}$ was measured as a function of the excess carrier density $\Delta n$ for each wafer over the temperature range of $0-270{ }^{\circ} \mathrm{C}$.

\section{RESULTS AND DISCUSSION}

The injection dependent lifetimes of the Ti-contaminated wafer taken from the $53 \%$ below the top of ingot, and the

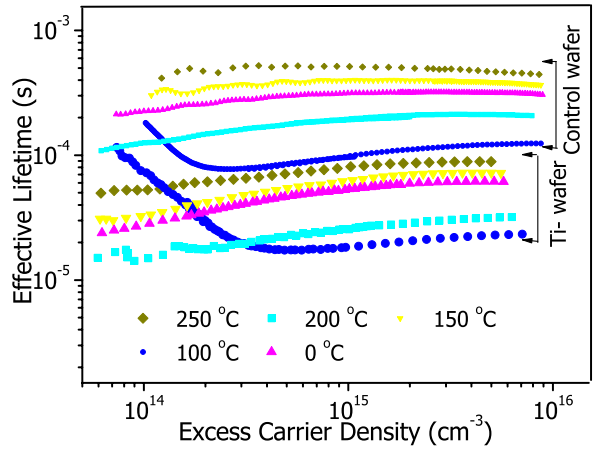

FIG. 1. (Color online) Injection dependent lifetime of a Ti-contaminated and control wafer.

control wafer, which was also from the similar position of the ingot, are depicted in Fig. 1 for temperatures of 0, 100, 150,200 , and $250{ }^{\circ} \mathrm{C}$. The effective lifetime of all four wafers and the control wafer was found to increase significantly at lower excess carrier densities, i.e., under low level injection (LLI). This is caused by minority carrier trapping for the temperatures below $100{ }^{\circ} \mathrm{C} .{ }^{12}$ However, for the temperature range above $100{ }^{\circ} \mathrm{C}$, the trapping effect was removed since the traps become filled with thermally generated carriers. ${ }^{12,13}$

The effective lifetime of the Ti-contaminated wafer was found to be only about seven times less than the effective lifetime of the control wafer taken from a similar position in the ingot, as depicted in Fig. 1. Hence the lifetime associated with the Ti defect $\tau_{\mathrm{Ti}}$ was calculated ${ }^{3}$ by

$$
\frac{1}{\tau_{\mathrm{Ti}}}=\frac{1}{\tau_{\text {Ti-wafer }}}-\frac{1}{\tau_{\text {control }}},
$$

where $\tau_{\text {Ti-wafer }}$ is the overall lifetime measured on the Ticontaminated wafer and $\tau_{\text {control }}$ is the lifetime of the corresponding control wafer taken from a similar position in the ingot.

The Ti concentration $\left(N_{t}\right)$ was determined by measuring the Shockley-Read-Hall (SRH) capture time constant for holes $\left(\tau_{p 0}\right)$ by applying the simplified version of the SRH equation $^{14,15}$ to the measured lifetime data. However, as there are three different energy levels of interstitial $\mathrm{Ti}$ that may occur in silicon, it was essential to first make sure of the dominance of a particular energy level before the determination of $\tau_{p 0}(T)$. As will be shown, the double-donor energy level $\left(E_{V}+0.28\right)$ dominates recombination over the temperature range of $140-270{ }^{\circ} \mathrm{C}$.

The simplified version of SRH lifetime for a single defect without trap, i.e., $\Delta n=\Delta p$ can be written as ${ }^{3}$

$$
\tau_{\mathrm{SRH}}=\frac{\tau_{n 0}\left(p_{1}+p_{0}+\Delta n\right)+\tau_{p 0}\left(n_{1}+n_{0}+\Delta n\right)}{\left(n_{0}+p_{0}+\Delta n\right)},
$$

where $\tau_{n 0}$ and $\tau_{p 0}$ are the fundamental capture time constants for electrons and holes, $n_{1}=N_{C} \exp \left[\left(E_{c}-E_{t}\right) / k_{B} T\right]$ and $p_{1}$ $=N_{v} \exp \left[\left(E_{t}-E_{v}\right) / k_{B} T\right], N_{C}$ and $N_{V}$ are the effective densities of sates at the conduction and the valance band edge, $k_{B}$ is Boltzmann's constant, $n_{0}$ and $p_{0}$ are the electron and hole densities at thermal equilibrium, and $\Delta n$ is the excess carrier density. For a $p$-type wafer $n_{0}$ can be neglected in comparison to $p_{0}$ and $\Delta n$, giving 


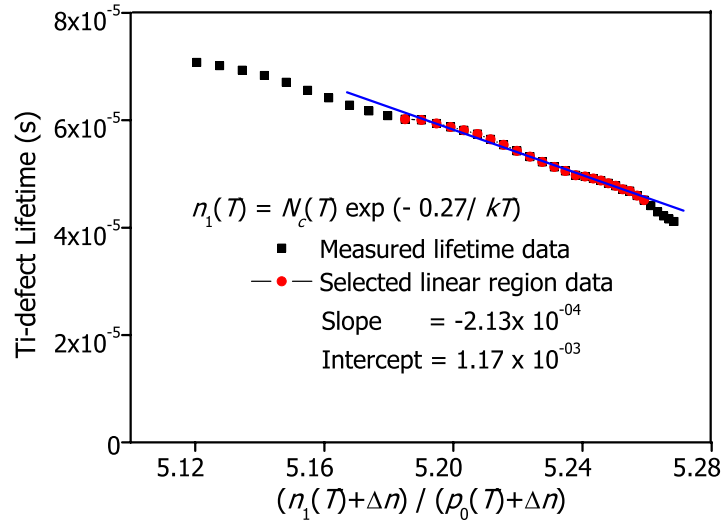

FIG. 2. (Color online) Lifetime of Ti-defect wafers vs $\left(n_{1}+\Delta n\right) /\left(p_{0}+\Delta n\right)$ for the donor level defect at $200{ }^{\circ} \mathrm{C}$.

$$
\tau_{\mathrm{SRH}}=\frac{\tau_{n 0}\left(p_{1}+p_{0}+\Delta n\right)+\tau_{p 0}\left(n_{1}+\Delta n\right)}{\left(p_{0}+\Delta n\right)} .
$$

\section{A. Defect energy level in upper band gap half (acceptor or donor)}

Now both the acceptor $\left(E_{C}-0.08\right)$ and donor $\left(E_{C}\right.$ -0.27) levels for interstitial Ti lie in the upper band gap half, making $p_{1}(T) \ll p_{0}(T)$ for all temperatures. In such situations Eq. (3) can be further simplified to

$$
\tau_{\mathrm{SRH}}=\tau_{n 0}+\tau_{p 0} \frac{\left(n_{1}+\Delta n\right)}{\left(p_{0}+\Delta n\right)}
$$

Thus, if all inherent assumptions are valid, the slope of a plot of $\tau_{\mathrm{SRH}}$ versus $\left(n_{1}+\Delta n\right) /\left(n_{1}+\Delta n\right)$ gives $\tau_{p 0}$ and the intercept gives $\tau_{n 0}$. Such plots of the measured data with $n_{1}$ for either donor or acceptor energy levels had negative slopes when $T$ was in the range of $140-270{ }^{\circ} \mathrm{C}$. Figure 2 provides an example at $200{ }^{\circ} \mathrm{C}$ for a Ti-wafer when the donor level is considered. Since $\tau_{p 0}$ cannot be negative, this observation rules out the dominance of energy levels in the upper band gap.

\section{B. Defect in lower band gap half (double donor)}

If instead, the dominant defect is the double-donor defect that lies in the lower half of the band gap, $n_{1}$ is negligible and Eq. (3) becomes

$$
\tau_{\mathrm{SRH}}=\frac{\tau_{n 0}\left(p_{1}+p_{0}+\Delta n\right)+\tau_{p 0}(\Delta n)}{\left(p_{0}+\Delta n\right)}
$$

and when $\Delta n$ is sufficiently less than $p_{0}$,

$$
\tau_{\mathrm{SRH}}=\frac{\tau_{n 0}}{p_{0}}\left(p_{1}+p_{0}\right)+\frac{\tau_{p 0}}{p_{0}} \Delta n
$$

Hence, the slope of a plot of $\tau_{\mathrm{SRH}}(\Delta n)$ gives $\tau_{p 0} / p_{0}$ and the intercept gives $\left(p_{1}+p_{0}\right) \tau_{n 0} / p_{0}$. The measured data and the plotted data for a Ti-wafer are depicted in Fig. 3 at $180{ }^{\circ} \mathrm{C}$, showing a region in which the assumptions above remain valid, i.e., $\Delta n \leq 3 \times 10^{14} \mathrm{~cm}^{-3}$. The slope of the measured lifetime of this sample for different temperatures is depicted

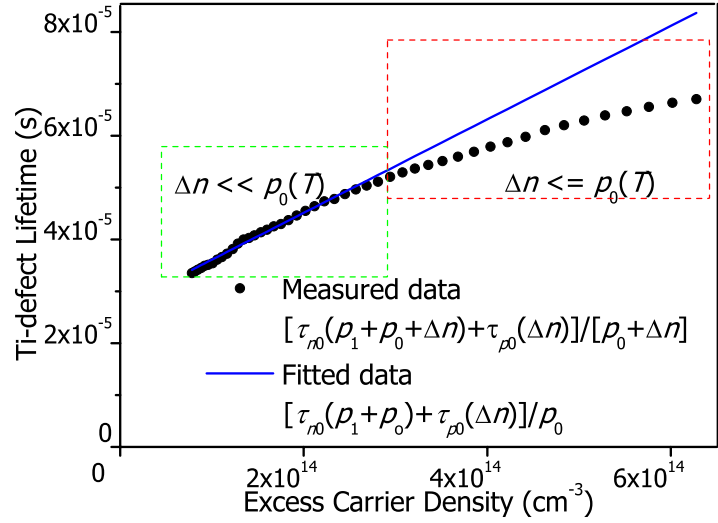

FIG. 3. (Color online) Measured lifetime data (Ti-wafer at $180{ }^{\circ} \mathrm{C}$ ) depicting linearity with the fitted model for LLI $\left[\Delta n \ll p_{0}(T)\right]$.

in Fig. 4, showing close agreement with the fitted model for the low injection region.

\section{Determination of $\mathrm{Ti}$ density for the double donor}

The concentration $(\mathrm{Nt})$ of interstitial Ti was determined by assuming that all recombination occurred through its double-donor defect energy level and hence at all temperatures, ${ }^{3}$

$$
N_{T}=\frac{1}{\tau_{p 0} v_{\mathrm{th}} \sigma_{p}}=\frac{1}{\text { slope } \times p_{0} v_{\mathrm{th}} \sigma_{p}},
$$

where the "slope" represents the slope of a plot of $\tau_{\mathrm{SRH}}(\Delta n)$ for low injection and the calculation does not require knowledge of $\sigma_{n}$. The calculations were made using $\sigma_{p}(T)=1.5$ $\times 10^{-16} \exp (-0.036 / k T)\left(\mathrm{cm}^{2}\right)$, as determined for the double-donor level, ${ }^{2}$ and the models for $v_{\mathrm{th}}(T)$ and $p_{0}(T)$ given by Green ${ }^{16}$ and Rein, ${ }^{3}$ respectively. $N_{t}$ is plotted in Fig. 5 , where the uncertainties result from the error in lifetime and in the least-squares linear fits. It depicts a constant $N_{t}$ at all temperatures, which supports the technique and the choice of the defect energy level's parameters $\left[E_{t}\right.$ and $\left.\sigma_{p}(T)\right]$. Note that below $140{ }^{\circ} \mathrm{C}$, there was no clear impact of the Ti-related energy level on the effective lifetime due to lower values of $n_{1}$ and $p_{1}$ and to carrier trapping. Note that Rein ${ }^{3}$ also concluded that the level in the lower half of the band gap (which is the double donor) is dominant for recombina-

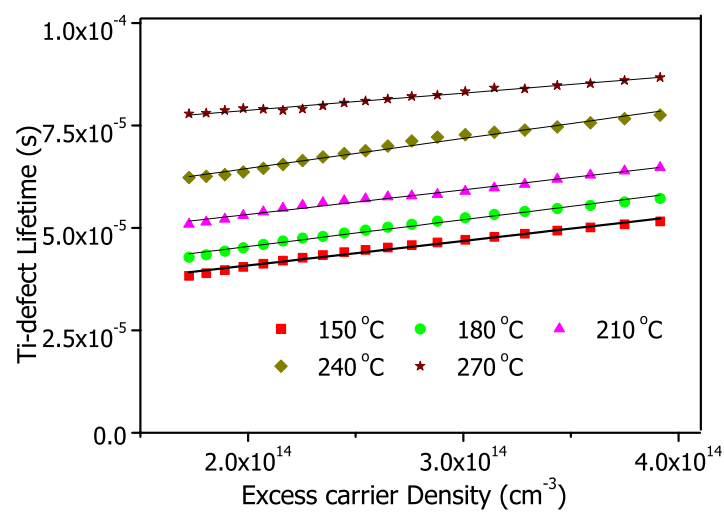

FIG. 4. (Color online) Measured carrier lifetime data showing consistency of Ti-wafer with fitted model for different temperatures. 


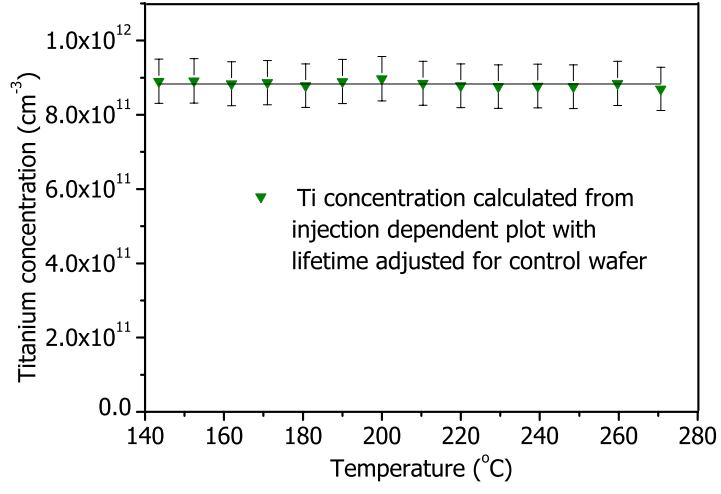

FIG. 5. (Color online) Measured value of the Ti concentration after lifetime data corrected with the control wafer data.

tion in $p$-type silicon by a combination of TDLS and IDLS.

\section{Determination of electron capture cross section}

The intercept on the lifetime axis of the plot of $\tau_{\text {eff }}(\Delta n)$ gives $\left(p_{1}+p_{0}\right) \tau_{n 0} / p_{0}$ according to Eq. (6). Hence, $\tau_{n 0}(T)$ can be determined using $p_{1}(T)=N_{v}(T) \exp \left(-\Delta E_{t} / k_{B} T\right.$, $)$, the energy level of the double donor and $p_{0}(T)$. With values of $\tau_{n 0}(T), N_{T}$, and $v_{\text {th }}(T)$, the $T$-dependent value of $\sigma_{n}(T)$ was calculated and is plotted in Fig. 6.

The $T$-dependent trends in $\sigma_{n}(T)$ associated with various capture mechanisms were listed by Rein. ${ }^{3}$ Of these mechanisms, only multiphonon emission (MPE) due to the deep centers ${ }^{17}$ yields an increase in $\sigma_{n}(T)$ with $T$, consistent with the measured data in Fig. 7. This suggests that the following mechanisms do not contribute significantly to electron capture at an interstitial Ti defect: photon emission and classical Auger, which have no $T$ dependence, and excitonic Auger capture due to deep centers, cascade capture, and two-stage cascade capture due to the deep coulomb attractive centers, ${ }^{18}$ all of which decrease with temperature. The electron capture cross section associated with MPE follows the equation ${ }^{17}$

$$
\sigma_{n}(T)=\sigma_{\infty} \exp \left(\frac{-E_{\infty}}{k_{B} T}\right),
$$

where $\sigma_{\infty}$ is the $T$-independent prefactor and $E_{\infty}$ is known as activation energy and where $\sigma_{\infty}=9.01 \times 10^{-14} \mathrm{~cm}^{2}$ and $E_{\infty}$ $=0.107 \pm 0.01 \mathrm{eV}$ provide the best fit to the experimental

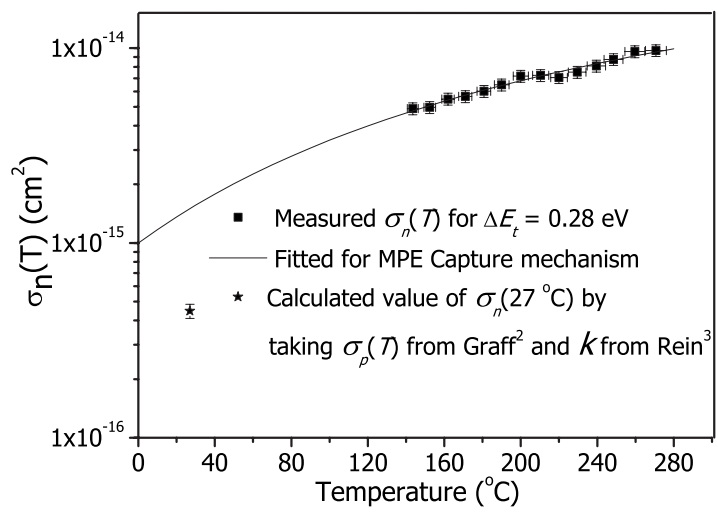

FIG. 6. Electron capture cross section for Ti fitted with MPE capture.

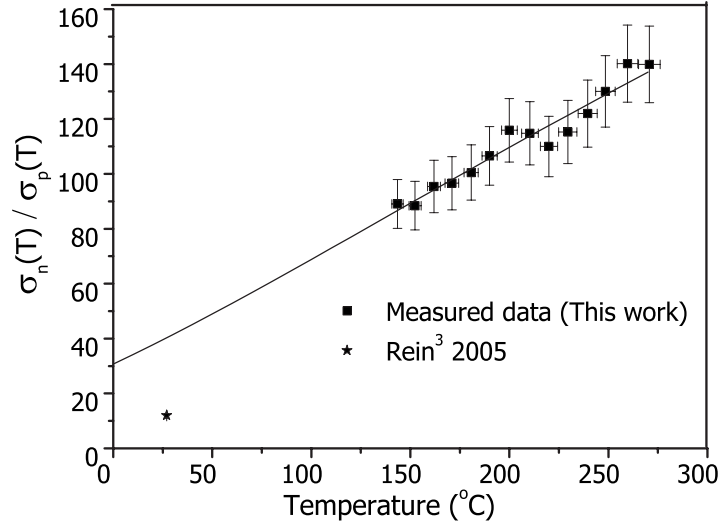

FIG. 7. Temperature dependence of carrier capture cross section ratio for double-donor level of $\mathrm{Ti}$ in silicon.

data (plotted in Fig. 7). Equation (8) and these best-fit parameters yield $\sigma_{n}(300 \mathrm{~K})=1.5 \times 10^{-15} \mathrm{~cm}^{2}$, which is 3.4 times higher than when calculated from $k$ determined by Rein $^{3}$ using $\sigma_{p}(300 \mathrm{~K})$ from Graff. ${ }^{2}$

$T$-dependent values of the capture cross section ratio $k(T)$ have also been determined and are plotted in Fig. 8. The capture cross section ratio was found to increase with temperature because the activation energy for hole capture is less than the activation energy for electron capture, even though both exhibit the same capture mechanism. The $T$-dependent trend of $k$ gives the value of $40.4 \pm 4$ at $27{ }^{\circ} \mathrm{C}$, which is 3.4 times higher than the value determined by Rein. ${ }^{3}$

\section{E. Determination of segregation coefficient}

Finally, a lower limit to the segregation coefficient of $\mathrm{Ti}$ in directionally cast multicrystalline silicon was determined by fitting the Scheil equation ${ }^{19,20}$ to the values of $N_{T}$ along the ingot length as stated in Eq. (9),

$$
C_{S}=k_{\mathrm{eff}} C_{0}\left(1-f_{S}\right)^{k_{\mathrm{eff}}-1} .
$$

Here, $C_{s}$ is the concentration of impurities in the solid phase, which is equal to $N_{t}$ determined in this work, $C_{0}$ is the initial concentration in the liquid, and $f_{s}$ is the fraction of solid formed. Ti concentrations on all four wafers were calculated by using Eq. (7). As there was no control for the wafers taken from the position $14 \%, 37 \%$, and $81 \%$ of the ingot, the non-

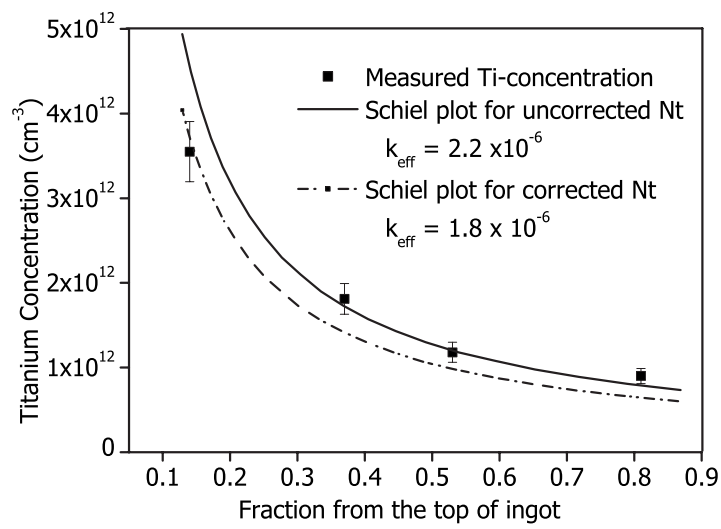

FIG. 8. Ti concentration for different positions within the ingot plotted with Scheil equation with different $k_{\text {eff }}$. 
corrected value of $N_{t}$ has been applied to calculate the segregation coefficient, which gives its lower limit. Furthermore, there are several preconditions required for the Scheil model to be valid: (a) diffusion in the solid is negligible; (b) local equilibrium is maintained at the solid-liquid interface and the curvature effect at the interface is neglected; (c) uniform liquid composition; and (d) equal solid and liquid densities. As these wafers are prepared by directional solidification and $\mathrm{Ti}$ is a slow diffuser, these conditions are believed to be approximately fulfilled in this case. The initial liquid concentration $C_{0}$ was $2.9 \times 10^{17} \mathrm{~cm}^{-3}$. The Ti concentrations determined in this work are shown in Fig. 8 as a function of the position in the ingot from which they came. Also shown is a fit of the Scheil equation. The value of the segregation coefficient that best fits the data is $2.2 \times 10^{-6}$ with $N_{t}$ calculated from the lifetime data, which was not corrected with the control wafer, and $1.8 \times 10^{-6}$ with $N_{t}$ calculated from the lifetime data adjusted with the control wafer. In the second case $N_{t}$ for one Ti-wafer was calculated and $N_{t}$ for the other three wafers was adjusted in the same proportion. Note that this is strictly only a lower limit on the segregation coefficient, as the solid concentrations were determined only for the double-donor defect energy level for interstitial Ti. If there are significant quantities of $\mathrm{Ti}$ present in other forms (for example, as the interstitial acceptor or donor, as substitutional $\mathrm{Ti}$, or as precipitates), then the segregation coefficient would be larger. This, in fact, could explain the deviation from the Scheil equation for the sample taken from the near the top of the ingot.

The segregation coefficient for Ti in single-crystal silicon ingot growth, which is assumed to represent the equilibrium segregation coefficient, has been reported variously as $1.8 \times 10^{-6},{ }^{21} 2 \times 10^{-6},{ }^{22}$ and $3.6 \times 10^{-6} .^{23}$ These values are very close to the value determined here. Assuming that there is an insignificant amount of $\mathrm{Ti}$ in forms other than the interstitial double donor, this would imply that the segregation of $\mathrm{Ti}$ in directionally solidified multicrystalline silicon proceeds essentially in equilibrium. Recent work has shown that the segregation coefficient of $\mathrm{Fe}$ in multicrystalline silicon is also close to the equilibrium value. ${ }^{24}$

\section{CONCLUSION}

Ti concentrations in multicrystalline silicon wafers were calculated by analysis of injection dependent carrier lifetime data for different temperatures, in conjunction with the published $T$-dependent model for $\sigma_{p}(T)$. Measured lifetime data for the temperature range $\left(140-270{ }^{\circ} \mathrm{C}\right)$ were used to calculate the $T$-dependent value of the electron capture cross section $\sigma_{n}(T)$ for the double-donor level of Ti in silicon. The $T$-dependent trend for $\sigma_{n}(T)$ best matched the MPE model for carrier capture and gave a comparable value with previ- ously published values of $\sigma_{n}(T)$ at room temperature when extrapolated. A lower limit for the segregation coefficient of $1.8 \times 10^{-6}$ for Ti in multicrystalline silicon was determined by using the Scheil model and indicated that segregation proceeds in equilibrium in this type of ingot growth.

\section{ACKNOWLEDGMENTS}

The authors thank Dr. Bart Geerligs of ECN, The Netherlands, for supplying the experimental samples and valuable suggestions. This work was funded by an Australian Research Council Linkage Grant between the Australian National University, SierraTherm Production Furnaces, and SunPower Corporation. D.H.M. is supported by an Australian Research Council fellowship.

${ }^{1}$ T. Buonassisi, A. A. Istratov, M. D. Pickett, M. Heuer, J. P. Kalejs, M. A. G. Hahn, B. L. Marcus, Z. Cai, and S. M. Heald, Prog. Photovoltaics 14, 513 (2006)

${ }^{2} \mathrm{~K}$. Graff, Metal Impurities in Silicon-Device Fabrication, 2nd ed. (Springer, Berlin, 1999), Vol. 24.

${ }^{3}$ S. Rein, Lifetime Spectroscopy: A Method of Defect Characterization in Silicon for Photovoltaic Applications (Springer, Berlin, 2005).

${ }^{4}$ T. Roth, M. Rüdiger, W. Warta, and S. W. Glunz, J. Appl. Phys. 104, 074510 (2008).

${ }^{5}$ L. J. Geerligs, P. Manshanden, G. P. Wyers, E. J. Øvrelid, O. S. Raaness, A. N. Waernes, and B. Wiersma, Proceedings of the 20th European Photovoltaic Solar Energy Conference, Barcelona, Spain, 2005 (WIPRenewable Energies, Münich, 2005), p. 619.

${ }^{6}$ L. J. Geerligs, P. Manshanden, I. Solheim, E. J. Øvrelid, and A. N. Waernes, Proceedings of the 21st European Photovoltaic Solar Energy Conference and Exhibition, Dresden, Germany, 2006 (WIP-Renewable Energies, Münich, 2005), p. 1285.

${ }^{7}$ S. Reggiani, M. Valdinoci, L. Colalongo, M. Rudan, G. Baccarani, A. D. Stricker, F. Illien, N. Felber, W. Fichtner, and L. Zullino, IEEE Trans. Electron Devices 49, 490 (2002).

${ }^{8}$ R. A. Sinton and A. Cuevas, Appl. Phys. Lett. 69, 2510 (1996).

${ }^{9}$ B. B. Paudyal, K. R. McIntosh, D. H. Macdonald, B. S. Richards, and R. A. Sinton, Prog. Photovoltaics 16, 609 (2008).

${ }^{10}$ D. B. M. Klaassen, Solid-State Electron. 35, 953 (1992).

${ }^{11}$ D. B. M. Klaassen, Solid-State Electron. 35, 961 (1992).

${ }^{12}$ D. Macdonald and A. Cuevas, Appl. Phys. Lett. 74, 1710 (1999).

${ }^{13}$ K. R. McIntosh, B. B. Paudyal, and D. H. Macdonald, J. Appl. Phys. 104, 084503 (2008)

${ }^{14}$ W. Shockley and W. T. J. Read, Phys. Rev. 87, 835 (1952).

${ }^{15}$ R. N. Hall, Phys. Rev. 87, 387 (1952).

${ }^{16}$ M. A. Green, J. Appl. Phys. 67, 2944 (1990).

${ }^{17}$ C. H. Henry and D. V. Lang, Phys. Rev. B 15, 989 (1977)

${ }^{18}$ R. M. Gibb, G. J. Rees, B. W. Thomas, B. L. H. Wilson, B. Hamilton, D. R. Wight, and N. F. Mott, Philos. Mag. 36, 1021 (1977).

${ }^{19}$ R. A. Brown and D. H. Kim, J. Cryst. Growth 109, 50 (1991).

${ }^{20}$ E. Scheil, Z. Metallkd. 34, 70-72 (1942).

${ }^{21}$ H. Lemke, in Semiconductor Silicon, edited by H. R. Huff, W. Bergholz, and K. Sumino (Electrochemical Society, Pennington, NJ, 1994), p. 695.

${ }^{22}$ J. R. Davis, A. Rohatgi, R. H. Hopkins, P. D. Blais, P. Rai-Choudhury, J. R. McCormick, and H. C. Mollenkopf, IEEE Trans. Electron Devices 27, 677 (1980).

${ }^{23}$ S. Pizzini, M. Acciarri, and S. Binetti, Phys. Status Solidi A 202, $2928-$ $2942(2005)$.

${ }^{24}$ R. Kvande, L. J. Geerligs, G. Coletti, L. Arnberg, M. D. Sabatino, E. J. Øvrelid, and C. C. Swanson, J. Appl. Phys. 104, 064905 (2008). 